\title{
Versión española del COPE-48: Evaluación del afrontamiento en pacientes psiquiátricos ambulatorios
}

\section{The Spanish version of the COPE-48: Assessing coping strategies in psychiatric outpatients}

\author{
Yolanda Martínez-Ortega
}

Centre de Salut Mental d'Adults, Fundació Althaia, Xarxa Assistencial Universitària de Manresa, Cataluña, España

Departamento de Psicología Clínica y de la Salud, Universitat Autònoma de Barcelona, Cataluña, España

Montserrat Gomà-i-Freixanet

Departamento de Psicología Clínica y de la Salud, Universitat Autònoma de Barcelona, Cataluña, España

Albert Fornieles-Deu

Departamento de Psicobiología y Metodología de las Ciencias de la Salud, Profesor Serra Húnter, Universitat Autònoma de Barcelona, Cataluña, España

Recibido (06/04/2017) Aceptado (23/09/2017)

Correspondencia: Centre de Salut Mental d'Adults, Fundació Althaia, Xarxa Assistencial Universitària de Manresa. C/ Dr. Llatjós s/n. 08243 Manresa (Barcelona). España. Tel. +349387421 12, Fax +349387593 09, Correo electrónico: ymartinezo@althaia.cat

Agradecimientos: Nuestro agradecimiento a todas las personas que desinteresadamente aceptaron participar en el proyecto. Agradecer la extraordinaria implicación de Mireia Ribas, Raquel Badrenas e Yrina Català en la fase de recogida de datos. También la colaboración inestimable de Anna Arnau por su ayuda en el análisis de los datos, así como a Carlos Aparicio por su implicación a lo largo del estudio. 


\title{
Resumen
}

Introducción: La utilización el COPE en contextos clínicos permite conocer las estrategias que utilizan las personas para afrontar situaciones estresantes y por ende incidir sobre ellas mediante tratamiento psicológico. Objetivo: presentar a la comunidad científica de habla hispana la versión española del COPE-48 junto con las propiedades psicométricas obtenidas en una muestra de personas adultas con psicopatología. Métodos: la muestra estuvo formada por 301 participantes (62.5\% mujeres). Resultados: la consistencia interna media de las escalas es de 0.81 . Nuestros resultados muestran un patrón diferencial en la utilización de las estrategias según el género. Conclusiones: la administración del COPE-48 en contextos clínicos es de gran utilidad práctica, puesto que permite conocer de modo rápido, fácil y preciso las estrategias más frecuentemente utilizadas por los pacientes para afrontar situaciones altamente demandantes. Dicho conocimiento permite intervenir mediante tratamiento psicológico para instaurar, potenciar o minimizar las estrategias necesarias para afrontar una determinada situación comprometida.

Palabras clave: Evaluación, COPE, Estrategias de afrontamiento, Trastornos mentales, Pacientes ambulatorios.

\begin{abstract}
Introduction: In clinical settings, the COPE questionnaire allows to assess the strategies people use to cope with stressful situations and therefore, if needed, to modify them through psychological treatment. Objective: The aim of this study is to offer to the Spanish-speaking scientific community the Spanish version of the COPE-48 along with the psychometric properties obtained in a sample of adults with psychopathology. Material and Methods: The sample consisted of 301 participants (62.5\% women). Results: The average internal consistency of the scales was 0.81 . Our results showed a gender pattern in the use of the coping strategies. Conclusions: In clinical settings, the administration of the COPE-48 allows a quick, easy and accurate assessment of the most frequently used strategies to cope with highly demanding situations. This knowledge makes possible to intervene with psychological treatment and whenever appropriate to establish, enhance or minimize the strategies needed to face difficult situations.
\end{abstract}

Keywords: Assessment, COPE, Coping skills, Mental disorders, Outpatients.

\section{Introducción}

Actualmente uno de los instrumentos más utilizados en la práctica clínica para la evaluación de los estilos de afrontamiento de las situaciones estresantes es el denominado Coping Orientation to Problems Experienced (COPE; Carver et al., 1989). La administración del COPE en contextos clínicos es de gran utilidad ya que nos permite conocer las estrategias que utilizan las personas con psicopatología para afrontar situaciones estresantes y por ende incidir sobre aquellas que se podrían potenciar o minimizar mediante tratamiento psicológico. Sin embargo, los datos de que disponemos en la actualidad respecto a las propiedades psicométricas de la versión española del COPE (Crespo y Cruzado, 1997) fueron obtenidos en una muestra de estudiantes. Dicha circunstancia conlleva un riesgo elevado de cometer errores en la interpretación de los resultados ya que las muestras clínicas y las de estudiantes difieren en aspectos tales como la edad, los años de escolarización e incluso el tipo de situaciones estresantes que deben afrontar (Kazdin, 1992; Muñiz et al., 2015), todo lo cual en conjunto afecta a su validez externa. Recientemente, nuestro equipo ha propuesto una nueva versión del COPE adecuada y validada para población psiquiátrica ambulatoria. Así pues, el objetivo de dicho artículo es presentar a la comunidad científica de habla hispana la versión española del COPE-48 (Martínez, Gomà-i-Freixanet y Fornieles, 2016) junto con las propiedades psicométricas obtenidas en una muestra de personas adultas con psicopatología. 


\section{Método}

\section{Participantes}

El estudio se llevó a cabo con pacientes que acudían a la Consulta de Psiquiatría de Atención Primaria de la Divisió de Salud Mental d'Althaia (Manresa, Barcelona). Los criterios de exclusión fueron: presentar problemas de comprensión oral o escrita, estar bajo los efectos de alguna sustancia tóxica, presentar un trastorno mental grave en estado agudo $\mathrm{y} / \mathrm{o}$ tener valores perdidos en el cuestionario. Accedieron a participar el $67.3 \%$ de los candidatos, y de éstos, excluimos el $1.74 \%$ debido a la presencia de valores perdidos en los cuestionarios. La muestra total final estuvo formada por 301 personas, mayoritariamente mujeres $(62.5 \%)$, con edades comprendidas entre 18 y 69 años $(M=44.11$ años; $D E=12.81)$ y estudios primarios $(47.5 \%)$ o secundarios (40.5\%). El $63.1 \%$ vivían en pareja, el $18.3 \%$ eran solteros/ as y el $16.3 \%$ separados/as o divorciados/as. Respecto a la nacionalidad, el 93\% eran de origen español. Los diagnósticos más prevalentes fueron los trastornos adaptativos (39.9\%) y los del estado de ánimo (35.9\%), seguidos a gran distancia de los trastornos de ansiedad (9.6\%).

\section{Instrumentos}

El COPE-48 (Martínez, et al., 2016) es un instrumento autoadministrado, de lápiz y papel, que consta de 48 ítems que evalúan, mediante una escala tipo Likert de cuatro puntos $(1=$ No lo hago nunca a $4=$ Lo hago muchas veces $)$, nueve estrategias de afrontamiento ante situaciones de estrés (véase Tabla 1). Puntuaciones elevadas en las escalas se interpretan como mayor uso de dichas estrategias. Dicho instrumento evalúa los estilos de afrontamiento desde una perspectiva disposicional.

Los datos sociodemográficos los recogimos mediante un cuestionario breve diseñado a tal efecto. El diagnóstico principal, establecido por el psiquiatra según criterios DSM-IV-TR (American Psychiatric Association, 2000), lo extrajimos de la historia clínica del paciente.

Tabla 1. Escalas del COPE-48 e items pertenecientes a cada escala

\begin{tabular}{|c|c|c|}
\hline Escala & $\mathrm{N}^{\mathrm{o}}$ & Ítem \\
\hline \multirow{9}{*}{$\begin{array}{l}\text { 1. Afrontamiento } \\
\text { activo del } \\
\text { problema }\end{array}$} & 1 & Concentro mis esfuerzos en hacer algo acerca de ello. \\
\hline & 2 & Elaboro un plan de acción. \\
\hline & 3 & Intento proponer una estrategia sobre qué hacer. \\
\hline & 4 & Me centro en hacer frente a este problema, y si es necesario dejo otras cosas un poco de lado. \\
\hline & 5 & Intento firmemente evitar que otras cosas interfieran con mis esfuerzos por hacer frente a esto. \\
\hline & 6 & Llevo a cabo una acción directa en torno al problema. \\
\hline & 7 & Dejo de lado otras actividades para concentrarme en esto. \\
\hline & 8 & Medito profundamente acerca de qué pasos tomar. \\
\hline & 9 & Hago lo que ha de hacerse, paso a paso. \\
\hline \multirow{4}{*}{$\begin{array}{l}\text { 2. Consumo de } \\
\text { alcohol o } \\
\text { drogas }\end{array}$} & 10 & Utilizo alcohol o drogas para hacerme sentir mejor. \\
\hline & 11 & Intento perderme un rato bebiendo alcohol o consumiendo drogas. \\
\hline & 12 & Bebo alcohol o tomo drogas para pensar menos en ello. \\
\hline & 13 & Utilizo alcohol o drogas para ayudarme a superarlo. \\
\hline \multirow{4}{*}{$\begin{array}{l}\text { 3. Centrarse en las } \\
\text { emociones y } \\
\text { desahogarse }\end{array}$} & 14 & Me altero y dejo que mis emociones afloren. \\
\hline & 15 & Me altero y soy realmente consciente de ello. \\
\hline & 16 & Dejo aflorar mis sentimientos. \\
\hline & 17 & Siento mucho malestar emocional y termino por expresar ese malestar. \\
\hline
\end{tabular}




\begin{tabular}{|c|c|c|}
\hline Escala & $\mathrm{N}^{\mathrm{o}}$ & Ítem \\
\hline \multirow{8}{*}{$\begin{array}{l}\text { 4. Búsqueda de } \\
\text { apoyo social }\end{array}$} & 18 & Intento conseguir consejo de alguien sobre qué hacer. \\
\hline & 19 & Hablo de mis sentimientos con alguien. \\
\hline & 20 & Hablo con alguien para averiguar más acerca de la situación. \\
\hline & 21 & Intento conseguir apoyo emocional de amigos o familiares. \\
\hline & 22 & Hablo con alguien que pueda hacer algo concreto acerca del problema. \\
\hline & 23 & Consigo el apoyo y comprensión de alguien. \\
\hline & 24 & Pregunto a personas que han tenido experiencias similares qué hicieron. \\
\hline & 25 & Hablo con alguien de cómo me siento. \\
\hline \multirow{4}{*}{ 5. Humor } & 26 & Me río de la situación. \\
\hline & 27 & Hago bromas sobre ello. \\
\hline & 28 & Bromeo sobre ello. \\
\hline & 29 & Hago bromas de la situación. \\
\hline \multirow{4}{*}{ 6. Religión } & 30 & Confío en Dios. \\
\hline & 31 & Busco la ayuda de Dios. \\
\hline & 32 & Intento encontrar alivio en mi religión. \\
\hline & 33 & Rezo más de lo habitual. \\
\hline \multirow{4}{*}{ 7. Negación } & 34 & Me digo a mi mismo: "esto no es real". \\
\hline & 35 & Me niego a creer que ha sucedido. \\
\hline & 36 & Finjo que no ha sucedido realmente. \\
\hline & 37 & Actúo como si nunca hubiera sucedido. \\
\hline \multirow{4}{*}{$\begin{array}{l}\text { 8. Refrenar el } \\
\text { afrontamiento }\end{array}$} & 38 & Me disuado a mí mismo de hacer algo demasiado rápidamente. \\
\hline & 39 & Demoro hacer algo acerca de ello hasta que la situación lo permita. \\
\hline & 40 & Me aseguro de no empeorar las cosas por actuar precipitadamente. \\
\hline & 41 & Me obligo a esperar el momento adecuado para hacer algo. \\
\hline \multirow{7}{*}{$\begin{array}{l}\text { 9. Aceptación y } \\
\text { crecimiento } \\
\text { personal }\end{array}$} & 42 & Acepto que esto ha pasado y que no se puede cambiar. \\
\hline & 43 & Intento verlo de manera diferente para hacerlo parecer más positivo. \\
\hline & 44 & Busco algo bueno en lo que está sucediendo. \\
\hline & 45 & Pienso en cómo podría manejar mejor el problema. \\
\hline & 46 & Acepto la realidad del hecho que ha sucedido. \\
\hline & 47 & Aprendo a vivir con ello \\
\hline & 48 & Aprendo algo de la experiencia. \\
\hline
\end{tabular}

\section{Procedimiento}

La fase de recogida de datos empezó en abril de 2013 y finalizó en diciembre de 2014. Psicólogas entrenadas administraron los cuestionarios mientras los participantes esperaban en la consulta con el psiquiatra. La participación fue voluntaria y no remunerada.

\section{Análisis de datos}

Para el análisis estadístico utilizamos el paquete SPSS (versión 22.0). Calculamos los descriptivos de las nueve escalas del
COPE-48 para la muestra total y para hombres y mujeres; así como la consistencia interna evaluada mediante el alfa de Cronbach para la muestra total y las diferencias de género mediante la prueba $t$ de Student.

\section{Resultados}

La consistencia interna media de esta nueva versión es de 0.81. La Tabla 2 muestra los valores descriptivos de las nueve escalas, tanto para la muestra total como para hombres y mujeres, los valores de consistencia interna para la muestra total, así como la comparación de medias entre géneros y la 
estimación del tamaño del efecto con la $d$ de Cohen. Ambos géneros obtuvieron puntuaciones significativamente diferentes en cuatro de las nueve escalas (Consumo de Alcohol/drogas, Centrarse en las Emociones, Religión y Negación).

Tabla 2. Medias, desviaciones estándar, coeficientes alfa de Cronbach y comparación de medias para las nueve escalas del COPE-48 ( $n=301)$

\begin{tabular}{|c|c|c|c|c|c|c|c|c|c|c|}
\hline \multirow[t]{2}{*}{ Escala } & \multicolumn{3}{|c|}{$\begin{array}{l}\text { Muestra total } \\
\quad(n=301)\end{array}$} & \multicolumn{2}{|c|}{$\begin{array}{l}\text { Hombres } \\
(n=113)\end{array}$} & \multicolumn{3}{|c|}{$\begin{array}{c}\text { Mujeres } \\
(n=188) \\
\end{array}$} & \multirow[b]{2}{*}{$p$} & \multirow[b]{2}{*}{ d de Cohen } \\
\hline & $M$ & $D E$ & $a$ & $M$ & $D E$ & $M$ & $D E$ & $t$ & & \\
\hline 1. Afrontamiento activo del problema & 2.48 & 0.51 & 0.84 & 2.45 & 0.49 & 2.50 & 0.52 & 0.842 & 0.400 & 0.10 \\
\hline 2. Consumo de alcohol o drogas & 1.34 & 0.68 & 0.94 & 1.49 & 0.75 & 1.25 & 0.62 & 2.813 & 0.005 & 0.35 \\
\hline 3. Centrarse en las emociones y desahogarse & 2.79 & 0.70 & 0.73 & 2.53 & 0.62 & 2.95 & 0.69 & 5.184 & $<0.001$ & 0.64 \\
\hline 4. Búsqueda de apoyo social & 2.52 & 0.74 & 0.90 & 2.43 & 0.71 & 2.57 & 0.75 & 1.592 & 0.113 & 0.19 \\
\hline 5. Humor & 1.72 & 0.76 & 0.91 & 1.83 & 0.80 & 1.65 & 0.73 & 1.955 & 0.052 & 0.24 \\
\hline 6. Religión & 1.86 & 0.91 & 0.90 & 1.59 & 0.81 & 2.02 & 0.93 & 4.183 & $<0.001$ & 0.49 \\
\hline 7. Negación & 1.88 & 0.68 & 0.69 & 1.73 & 0.60 & 1.97 & 0.72 & 3.025 & 0.003 & 0.36 \\
\hline 8. Refrenar el afrontamiento & 2.45 & 0.59 & 0.55 & 2.46 & 0.60 & 2.45 & 0.93 & 0.195 & 0.846 & 0.01 \\
\hline 9. Aceptación y crecimiento personal & 2.61 & 0.61 & 0.81 & 2.56 & 0.60 & 2.60 & 0.63 & 0.401 & 0.688 & 0.07 \\
\hline
\end{tabular}

\section{Discusión}

En el presente estudio, presentamos la versión española del COPE-48. Las estrategias de afrontamiento más utilizadas en nuestra muestra fueron las de Centrarse en las Emociones y Desahogarse, así como Aceptación y Crecimiento Personal y Búsqueda de Apoyo Social. Dichos resultados son similares a los hallados por otros autores (Gutiérrez et al., 2007; Lundqvist y Ahlström, 2006; Pozzi et al., 2015) en muestras clínicas que acudían a una consulta de salud mental en busca de apoyo y ayuda profesional.

La versión española del COPE-48 presenta una consistencia interna aceptable-buena, a excepción de la escala Refrenar el Afrontamiento. Sin embargo, hemos mantenido dicha escala por su utilidad clínica ya que es la única que permite evaluar el afrontamiento centrado en el autocontrol y el COPE-48 no dispone de otras escalas que aborden dicho estilo de afrontamiento.

En referencia a las diferencias de género, nuestros resultados apuntan en la misma dirección que los obtenidos en estudios previos, en los que las mujeres tienden a centrarse más en las emociones y a utilizar estrategias religiosas y de negación (Carver et al., 1989; Kallasmaa y Pulver, 2000) mientras que los hombres utilizan más el consumo de tóxicos (NolenHoeksema, 2004). Dichas diferencias podrían deberse al propio proceso de socialización o al tipo de situaciones estresantes que de manera diferencial se exponen hombres y mujeres (Matud, 2004).
Debemos mencionar que los datos recogidos han sido auto informados lo cual podría implicar ciertos sesgos debidos a una posible intencionalidad en las respuestas (e.g. deseabilidad social o magnificación de dificultades). Por otra parte, la muestra estaba formada mayoritariamente por personas de origen español lo cual podría mermar la generalización de los resultados a personas procedentes de otras culturas. Sin embargo, dicho estudio aporta datos de gran validez ecológica ya que la muestra utilizada está constituida por pacientes no seleccionados que acuden a una consulta psiquiátrica ambulatoria; a diferencia de los estudios realizados con muestras de estudiantes, que suelen estar formadas por jóvenes, que en su mayoría han estado expuestos a un número menor de situaciones estresantes y de menor gravedad, y que probablemente disponen de mayor resiliencia (Henrich et al., 2010; Lundqvist y Ahlström, 2006).

En resumen, proporcionamos datos de referencia de la versión española del COPE-48, diferenciados por género, de una amplia muestra ambulatoria de personas con psicopatología. Consideramos que la administración del COPE-48 en contextos clínicos y de investigación es, sin duda, de gran utilidad práctica ya que nos permite conocer de modo rápido, fácil y preciso las estrategias más frecuentemente utilizadas por los pacientes para afrontar situaciones altamente demandantes. En la práctica clínica, dicho conocimiento nos permite intervenir mediante tratamiento psicológico para en su caso instaurar, potenciar o minimizar las estrategias necesarias para afrontar una determinada situación comprometida. 


\section{Referencias}

American Psychiatric Association (2000). DSM-IV-TR: Diagnostic and Statistical Manual of Mental Disorders (4th Revised). Washington: American Psychiatric Association.

Carver, C.S., Scheier, M.F., y Weintraub, J. K. (1989). Assessing coping strategies: A theoretically based approach. Journal of Personality and Social Psychology, 56, 267-283. doi: http://dx.doi.org/10.1037/00223514.56.2.267

Crespo, M., y Cruzado, J.A. (1997). La evaluación del afrontamiento: adaptación española del cuestionario COPE con una muestra de estudiantes universitarios. Análisis y Modificación de Conducta, 23, 797-830.

Gutiérrez, F., Peri, J.M., Torres, X., Caseras, X., y Valdés, M. (2007). Three dimensions of coping and a look at their evolutionary origin. Journal of Research in Personality, 41, 1032-1053. doi: 10.1016/j.jrp.2007.01.006

Henrich, J., Heine, S.J., y Norenzayan, A. (2010). The weirdest people in the world? Behavioral and Brain Sciences, 33, 61-83. doi:10.1017/ S0140525X0999152X

Kallasmaa, T., y Pulver, A. (2000). The structure and properties of the Estonian COPE inventory. Personality and Individual Differences, 29, 881-894. doi: 10.1016/S0191-8869(99)00240-8

Kazdin A. E, editor. (1992). Methodological issues y strategies in clinical research. Washington DC: American Psychological Association. doi: http://dx.doi.org/10.1037/10109-000
Lundqvist, L.O, y Ahlström, G. (2006). Psychometric evaluation of the ways of coping questionnaire as applied to clinical and nonclinical groups. Journal of Psychosomatic Research, 60, 485-493. doi:10.1016/j. jpsychores.2005.08.019

Martínez Ortega, Y., Gomà-i-Freixanet, M., y Fornieles, A. (2016). The COPE-48: An adapted version of the COPE inventory for use in clinical settings. Psychiatry Research, 30, 808-814. doi: http://dx.doi. org/10.1016/j.psychres.2016.10.031

Matud, M.P. (2004). Gender differences in stress and coping styles. Personality and Individual Differences, 37, 1401-1415. doi:10.1016/j. paid.2004.01.010

Muñiz, J., Hernández, A., y Ponsoda, V. (2015). Nuevas directrices sobre el uso de los tests: Investigación, control de calidad y seguridad. Papeles del Psicólogo, 36, 161-173.

Nolen-Hoeksema, S. (2004). Gender differences in risk factors and consequences for alcohol use and problems. Clinical Psychology Review, 24, 981-1010.

Pozzi, G., Frustaci, A., Tedeschi, D., Solaroli, S., Grandinetti, P., Di Nicola M, y Janiri, L. (2015). Coping strategies in a sample of anxiety patients: Factorial analysis and associations with psychopathology. Brain and Behavior, 5. doi: 10.1002/brb3.351 